
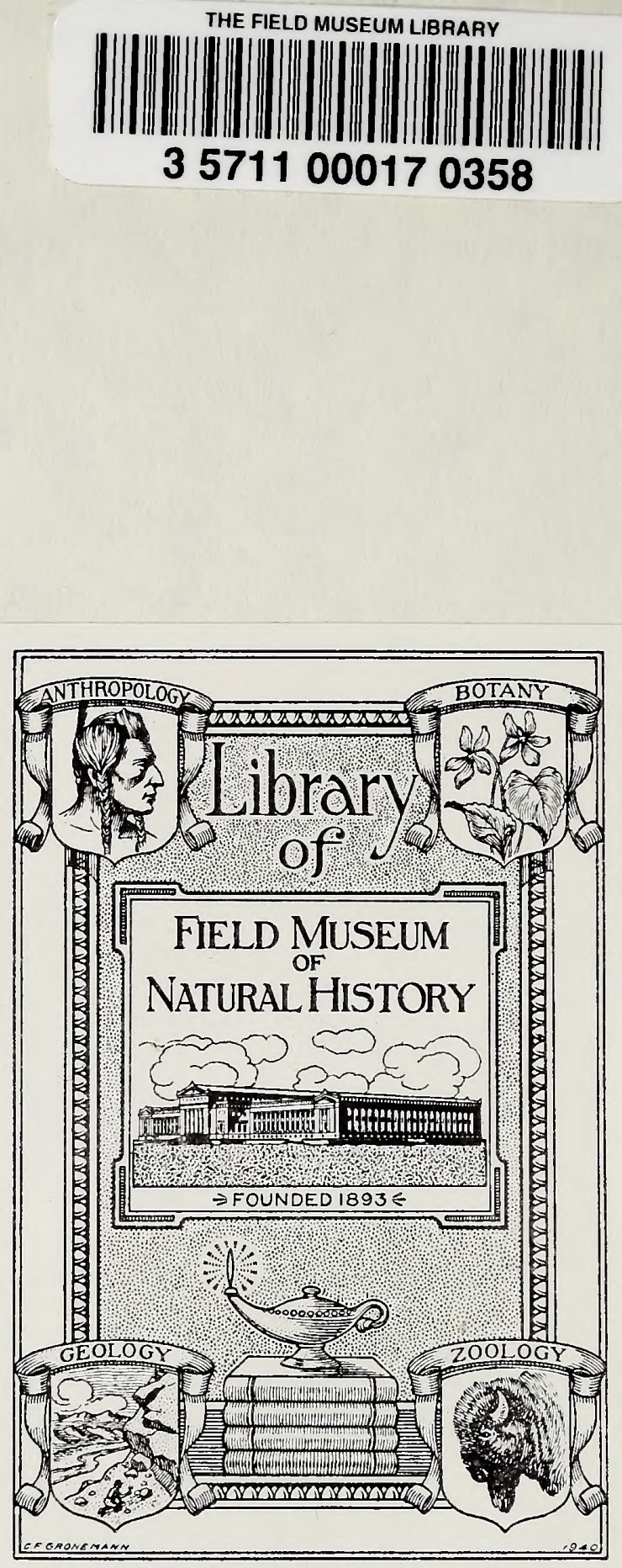


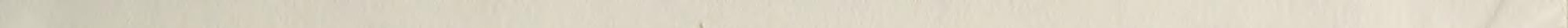


Digitized by the Internet Archive in 2013

http://archive.org/details/vegetationofchic00full 


\title{
The
}

Vegetation of the Chicago Region

An outline of some of the principal plant associations together with lists of their principal species

\author{
COMPILED BY \\ GEO. D. FULLER \\ Department of Botany, The University of Chicago
}

CHICAGO, ILLINOIS

1917 
$9 k$
157
156
1917

217365 


\section{THE VEGETATION OF THE CHICAGO REGION}

In this outline no attempt is made to treat exhaustively either the plant associations or the list of species composing them. Only the clearly defined associations easily accessible from the city of Chicago are included, more particular attention being given to those peculiar to this region. The names of weeds or ruderal plants are not included in the lists. For more detailed studies of these plant associations see Cowles, The plant societies of Chicago and vicinity, Geographic Soc. of Chicago, Bull. 2. I90I, and for the plants of the vernal flora see Cowles and Coulter, A flora for high schools, pp. I44. ill. New York: Amer. Book Co., I9I5.

\section{THE SAND DUNE AND UPLAND ASSOCIATIONS}

The following paragraphs outline the well-marked succession of plant associations upon the sand dunes of the Lake Michigan shores near Chicago and include several of the more typical associations of the forested morainic uplands. Following the outline is a list of many of the characteristic plants of these associations, the name of each species being preceded by a number or by numbers corresponding to those of the association or associations in which it chiefly occurs. For details regarding the vegetation of the sand dunes see Cowles, Bot. Gaz. 27:95 ff. I899. For evaporation and soil moisture conditions see Fuller, Bot. Gaz. 58:193-234. I914.

I. The beach association.-The vegetation of the beach is scanty, consisting principally of xerophytic annuals together with a few perennial herbs and shrubs.

2. The fore-dune associations.-This region of embryonic dunes is characterized by the sand reed grasses and such shrubs as the willow and the sand cherry together with the herbaceous plants of the beach. Well-developed fore-dunes may be seen at Lake Bluff, Ill., Miller and Dune Park, Ind., and Sawyer, Mich.

3. The cottonwood association. - This is notably the area of actively moving dunes with sparse vegetation. The rate of evaporation is very high, but the water content of the soil remains very constant at a short 
distance below the surface. The herbaceous and shrub vegetation of the previous associations persists with few additions. The dominant and almost the only tree species (Populus deltoides) gives its name to the association, but is occasionally replaced by the closely allied Populus balsamifera. The germination and development of these trees are dependent upon such moist areas as pannes and the margins of ponds. (See Fuller, Trans. Ill. Acad. Sci. 5: 137-I43. I9I2.)

The cottonwood owes its successful establishment and permanence upon the moving dunes to its great powers of vegetative activity which is seen in the production of adventitious roots from the buried trunk and branches and also in the development of adventitious shoots from its uncovered roots.

4. The pine dune association.-Soon after the fixation of the dunes there develops an association succeeding the cottonwood and characterized by evergreens. Among the dominant plants are species of pine, juniper, and arbor vitae, together with an undergrowth in which the heath family is well represented in species of Arctostaphylos, Pyrola, Gaultheria, etc. The herbaceous and shrub vegetation becomes much more abundant and is composed of species quite different from those in the former association. Humus formation proceeds, but the water content of the soil is less than in the previous association on account of the greater demands made upon it by the increasing amount of vegetation. The evaporating power of the air is, however, much less, and hence permits the development of seedlings of oak and other trees which dominate the succeeding association. The pine association is short-lived, but the undergrowth finally shows very decidedly mesophytic forms, especially upon the north face of slopes. This association was formerly well developed over much of the area between Buffington and Gary, Ind., where a few remnants are still to be seen. It occurs between Miller and Dune Park, Ind., and in a modified form at Lake Bluff, Ill., and Sawyer, Mich.

5. The black oak dune association.-This forest association consists of a somewhat open and often almost pure stand of Quercus velutina with an undergrowth of rather xerophytic herbs and shrubs. The evergreen forms of the previous association disappear and are replaced by such shrubs as species of Vaccinium, Rhus, Prunus, Sassafras, Viburnum, etc., while noticeable among the herbs are species of Solidago, Asclepias, Opuntia, Smilacina, etc. Soil moisture and evaporation conditions differ but little from those of the previous association, and the increase of humus in the soil is very slow. The association apparently persists for a much longer time than any of its predecessors, and is seen exten- 
sively developed near Miller, Ind., but is common throughout the entire dune region of Indiana and southern Michigan.

6. The mesophytic oak forest association.-Upon the sand dunes this gradually develops from the preceding by the coming in of other and more mesophytic tree species, notably such oaks as $Q$. alba and Q. rubra. It usually begins in sheltered depressions where the undergrowth is often very completely mesophytic before a marked change has taken place in the tree vegetation.

Upon morainic uplands this association differs but little from that found upon the dunes except for the presence of the hickories and the frequent occurrence of the bur oak, Q. macrocarpa. Depressions in clay upland within this forest are often characterized by the presence of the swamp white oak, Q. bicolor, or by the pin oak, Q. palustris. Corylus americana and Cormus paniculata are often the most abundant shrubs of the oak uplands.

Oak forests of this type are to be seen upon the sand dunes at Bridgman and Sawyer, Mich., and in less perfect development at Dune Park and Furnessville, Ind. The uplands at Smith, Ind., at Glencoe, Lake Bluff, Palos Park, New Lenox, and Starved Rock Park, Ill., show developments of oak-hickory forest of the same general type but with certain minor differences of detail.

7. The climax mesophytic forest.-This occurs, not only upon the sand dunes and the morainic uplands, but also upon all soils throughout the northeastern United States and the adjacent parts of Canada. It is dominated in this region by the beech and sugar maple, which often form more than 80 per cent of the tree growth. The hemlock is absent from the uplands of the Chicago region, but is seen upon the adjacent sand dunes of Michigan and in many other localities to the east and north. Perhaps the most conspicuous difference between this association as it occurs upon a clay upland and the corresponding forest upon adjacent sand dunes is the greater richness of the fern flora in the former locality.

The evaporating power of the air in the lower strata of the forest is very low when compared with that of other associations, and at the same time the soil moisture remains constantly well above the wilting coefficient.

This forest is to be seen upon the sand dunes at Sawyer, Mich., and upon the uplands at Three Oaks, Mich., Smith and Otis, Ind.

8. Sandy swamp associations.-From the nature of the recent geologic history of the region a series of old lagoons has been included in the dune area, and these now exist either as small shallow lakes or 
ponds or as sandy swamps in various stages of development. No attempt will be made to analyze the various stages in the plant succession from the open water to the forest, but some of the most characteristic plants of the later stages are included in the list. The plants of the open water and earlier swamp stages may be found listed under aquatic associations. The earliest shrubs are commonly the willows and they appear to be succeeded by a forest in which the poplars, pines, red maple, and sour gum are important members. Such transitional associations from the swamp to the dune find their best expression at Miller and Dune Park, Ind.

\section{Dune and Upland Plants ${ }^{\top}-$ Trees and Shrubs}

(7) Taxus canadensis Marsh., American yew.

(4) Pinus Strobus L., white pine.

(4*) " Banksiana Lamb., Jack pine.

(7*) Tsuga canadensis Carr., hemlock.

(4) Thrija occidentalis L., Arbor vitae.

(4*) Juniperus communis L., common juniper.

(4) " virginiana L., red cedar.

(4) " horizontalis Moench., prostrate juniper.

$(3,4)$ Salix longifolia Muhl., long-leaved willow.

$(2,3,4)$ " syrticola Fernald, glandular willow.

$(2,3,4)$ " glaucophylla Bebb, glaucous willow.

$(3,4,5)$ " humilis Marsh., prairie willow.

(8) Populus tremuloides Michx., American aspen.

(8) " grandidentata Michx., large-toothed aspen.

(3) " balsamifera L., balsam poplar.

$\left(3^{*}\right)$ " deltoides Marsh., cottonwood.

(5) Myrica asplenifolia L., sweet fern.

$(8,7)$ Juglans cinerea L., butternut.

(6) Carya ovata (Mill) K. Koch., shag-bark hickory (u).

(6) " cordiformis (Wang.) K. Koch., bitter-nut hickory (u).

(6*) Corylus americana Walt., hazelnut (u).

(6) Ostrya virginiana K. Koch., hop-hornbeam (u).

(6) Carpinus caroliniana Walt., water-beech or blue beech $(u)$.

( $\left.7^{*}\right)$ Fagus grandifolia Ehrh., beech.

(6*) Quercus alba L., white oak.

$\left(6^{*}\right)$ " macrocarpa Michx., bur oak $(u)$.

(6) " imbricaria Michx., shingle oak $(u)$.

$(5,6)$ " Muhlenbergii Engelm., chestnut oak.

$\left(6^{*}, 7\right) \quad$ " rubra L., red oak.

$\left(5^{*}, 6\right) \quad$ " velutina Lam., black oak.

$(6,7)$ Ulmus fulva Michx., slippery elm.

$(6,7,8)$ " americana L., American elm.

The numerals preceding the names indicate the association or associations, outlined in the foregoing paragraphs, in which the plant is usually found. Dominant or very abundant species are indicated by an asterisk $(*)$, while species commonly found upon the uplands only are indicated by $(u)$ following the common name. 
(5) Celtis occidentalis pumila Muhl., dwarf hackberry.

(7) Liriodendron Tulipifera L., tulip tree.

(7) Asimina triloba Dunal., American papaw.

$\left(5^{*}, 6\right)$ Sassafras variifolium (Salisb.) Ktze., sassafras.

(7) Benzoin aestivale (L.) Nees., spice-bush.

(6) Ribes Cynosbati L., prickly gooseberry $(u)$.

$(6,7)$ Hamamelis virginiana L., witch-hazel.

$(4,5)$ Rosa acicularis Lindl., wild rose.

$(4,5)$ " blanda Ait., wild rose.

$(4,5)$ " humilis Marsh., wild rose.

(5) Amelanchier canadensis Medic., service-berry or shad-bush.

(8) Pyrus arbutifolia L., chokeberry.

(5) Prunus pennsylvanica L., pin cherry.

(5) " virginiana L., chokecherry.

(7) “ serotina Ehrh., wild black cherry (u).

$\left(2^{*}, 3\right)$ " pumila L., sand cherry.

(5) Ptelea trifoliata L., hop-tree.

$(4,5)$ Rhus typhina L., staghorn sumach.

$(4,5)$ " copallina L., dwarf sumach.

$(5,6)$ " glabra L., smooth sumach.

$(3,4,5,6)$ " Toxicodendron L., poison-ivy.

$(4,5)$ " canadensis Marsh., aromatic sumach.

(7) Evonymus obovatus Nutt., strawberry bush.

$(3,4,5)$ Celastrus scandens L., climbing bittersweet.

$\left(7^{*}\right)$ Acer saccharum Marsh., sugar maple.

(8) “ rubrum L., red maple.

(5) Ceanothus americanus L., New Jersey tea.

(4, 5) Vitis vulpina L., wild grape.

$(4,5)$ " cordifolia Michx., wild grape.

$(4,5)$ " aestivalis Michx., summer grape.

$(4,5)$ " bicolor Le Conte, summer grape.

(4, 5, 6, 7) Psedera quinquefolia (L.), Greene, Virginia creeper.

$(6,7)$ Dirca palustris L., leatherwood.

$(4,6,7)$ Tilia americana L., basswood or American linden.

$(6,7)$ Cornus florida L., flowering dogwood.

$(4,8)$ " stolonifera Michx., red-osier dogwood.

(7) “ circinata L'Her., round-leaved dogwood.

(6) “ paniculata L'Her., panicled dogwood.

(7) “ alternifolia L., alternate-leaved dogwood (u).

(8) Nyssa sylvatica Marsh., sour gum.

(4*) Arctostaphylos Uva-ursi (L.) Spreng., bearberry.

(5) Gaylussacia baccata C. Koch., huckleberry.

(5) Vaccinium pennsylvanicum Lamb., blueberry.

(5) " vacillans Kalm., late blueberry.

$\left(5^{*}\right)$ Diervilla Lonicera Mill., bush honeysuckle.

(6) Lonicera canadensis Marsh., American fly honeysuckle (u).

(5) Viburnum acerifolium L., maple-leaved haw.

(7) Sambucus racemosa L., red-berried elder.

(7) " canadensis L., common elder. 


\section{Dune and Opland Plants-Herbaceous}

(5) Pteris aquilina L., common brake.

(7) Adiantum pedatum L., maidenhair fern.

(7) Asplenium acrostichoides Sw., golden spleenwort $(u)$.

(7) " angustifolium Michx., narrow-leaved spleenwort $(u)$.

(7) Polystichum acrostichoides Schott., Christmas fern.

(7) Aspidium marginale Sw., marginal shield fern.

(7) " spinulosum Sw., spinulose shield fern.

(7) " noveboracense (L) Sw., New York fern $(u)$.

(7) " Goldianum Hook., Goldie's fern $(u)$.

(8) " Thelypteris Sw., swamp shield fern.

(6) Osmunda Claytoniana L., interrupted fern $(u)$.

$(6,7)$ Botrychium virginianum Sw., rattlesnake fern.

(3) Equisetum arvense L., common horsetail.

(3) " hyemale L., scouring-rush.

(8) " variegatum Schleich., variegated horsetail.

(4*) Andropogon scoparius Michx., blue stem grass.

(5) Panicum virgatum L., switch grass.

(5) " dichotomum L., panic grass.

$(5,6)$ " spp., panic grass.

$\left(2^{*}, 3\right)$ Calamovilfa longifolia Hack., sand reed-grass.

$\left(2^{*}, 3\right)$ Ammophila arenaria Link., Marram grass.

$(2,3,4)$ Elymus canadensis L., rye-grass.

(5) Koeleria cristata Pers.

(5) Sphenopholis obtusata Scribn.

(5) Stipa spartea Trin., porcupine grass.

(5) Aristida tuberculosa Nutt., triple-awned grass.

(5) Festuca octoflora Walt.

(7) Hystrix patula Moench., bottle-brush grass $(u)$.

(5) Carex Muhlenbergii Schkuhr., Muhlenberg's sedge.

(5) " pennsylvanica Lam., Pennsylvania

(5) " umbellata Schkuhr., sedge.

$(6,7)$ " eburnea Boott., sedge.

(7) " laxiflora Lam., sedge.

(7) " plantaginea Lam., broad-leaved sedge $(u)$.

(5) Cyperus Schweinitzii Torr.

(5) " filiculmis Vahl.

(6) Luzula campestris (L) DC., wood rush.

(7) Arisaema triphyllum Schott., Indian turnip.

(5) Tradescantia virginiana L., spiderwort.

(8) Juncus balticus littoralis Willd., sand-rush.

(8) Tofieldia glutinosa Pers., false asphodel.

(8) Lilium philadelphicum L., wild orange lily.

(7) Allium tricoccum Ait., wild leek $(u)$.

(7) Erythronium americanum Ker., yellow adder's tongue $(u)$.

(7) " albidum Nutt., white adder's tongue or dog's-tooth violet $(u)$.

(6) Maianthemum canadense Desf., wild lily-of-the-valley.

$(4,5)$ Smilacina stellata (L.) Desf., false Solomon's seal.

$(4,5) \quad$ " racemosa (L.) Desf., false Solomon's seal. 
(7) Uvularia grandiflora Sm., bellwort.

$(5,6)$ Polygonatum commutatum Dietr., Solomon's seal.

(6) " biflorum (Walt.) Ell., Solomon's seal.

(6) Trillium recurvatum Beck., wake robin $(u)$.

(6) " sessile L., wake robin $(u)$.

(6) " declinatum (Gray) Gleason, white trillium $(u)$.

$\left(7^{*}\right)$ " grandiflorum Salisb., great white trillium.

(8) Aletris farinosa L., colic root.

(7) Smilax herbacea L., carrion flower.

(7) " hispida Muhl., cat-brier.

(8) Hypoxis hirsuta Coville, star-grass.

$(6,7)$ Cypripedium parviflorum, yellow lady's slipper.

(6) Orchis spectabilis L., showy orchis $(u)$.

(7) Pilea pumila (L.) Gray, clearweed (u).

(7) Parietaria pennsylvanica Muhl., pellitory.

(7) Laportea canadensis Gaud., wood nettle.

(5) Comandra umbellata Nutt., bastard toadflax.

(5) Polygonella articulata Meisn.

$(2,3)$ Cycloloma atriplicifolium Coult., winged pigweed.

$(2,3)$ Corispermum hyssopifolium L., bugseed.

$(3,5)$ Salsola Kali tenuifolia L., Russian thistle.

(7) Phytolacca decandra L., poke weed.

(5) Rumex Acetosella L., field sorrel.

(5) Arenaria stricta Michx., sandwort.

(6) " lateriflora L., lateral-flowered sandwort.

(7) Asarum canadense L., wild ginger (u).

(6) Silene stellata (L.) Ait., starry campion (u).

(6) Claytonia virginica L., spring beauty $(u)$.

(7) Thalictrum dioicum L., early meadow rue $(u)$.

(7) Anemonella thalictroides (L.) Spach., rue anemone (u).

(7) Hepatica acutiloba DC., hepatica.

(7) " triloba Chaix., hepatica.

(5) Anemone cylindrica Gray, anemone.

(5) " canadensis L., anemone.

(6) " quinquefolia L., wood anemone (u).

(6) " virginiana L., anemone.

(5) Aquilegia canadensis L., columbine.

(7) Actaea alba Mill., white baneberry.

(7) Isopyrum biternatum (Raf.) T. \& G., false rue anemone.

$(6,7)$ Podophyllum peltatum .., May apple.

(7) Caulophyllum thalictroides Michx., blue cohosh.

(7) Sanguinaria canadensis L., bloodroot.

(7) Stylophorum diphyllum Nutt., celandine poppy.

(7) Dicentra Cucullaria Bernh., Dutchman's breeches.

(7) " canadensis Walp., squirrel's corn.

$\left(I^{*}, 2,3\right)$ Cakile edentula Hook., sea rocket.

$\left(5^{*}\right)$ Arabis lyrata, rock cress.

(6) Dentaria laciniata Muhl., toothwort (u).

(7) Mitella diphylla L., miterwort. 
(6) Fragaria vesca L., wood strawberry $(u)$.

(6) Geum virginianum L., avens.

(6) Agrimonia spp., agrimony.

(I, 2) Potentilla Anserina L., goose-grass.

(8) " fruticosa L., shrubby cinquefoil.

(6) “ canadensis L., Canada cinquefoil $(u)$.

(8) Spiraea latifolia Borkh., meadow-sweet.

(8) “ tomentosa L., steeple bush.

(6) Desmodium canadensis DC., tick-trefoil $(u)$.

(6) " spp., tick-trefoil.

$(5,6)$ Baptisia leucantha T. \&. G., false indigo.

(5*) Lupinus perennis L., lupine.

(4) Petalostemum purpureum Rydb., prairie clover.

(5) Tephrosia virginiana Pers., hoary pea.

(5) Lespedeza capitata Mich., bush-clover.

(5) " violacea Pers., bush-clover.

$\left(\mathrm{I}, 2^{*}\right)$ Lathyrus maritimus Bigel., beach-pea.

(6) " venosus Muhl., vetchling $(u)$.

(6) " ochroleucus Hook., cream-colored vetchling $(u)$.

$(2,3)$ Strophostyles helvola Britton, beach-bean.

(6) Oxalis violacea L., violet wood sorrel $(u)$.

(6) " stricta L., yellow wood sorrel $(u)$.

$(6,7)$ Geranium maculatum L., wild geranium.

(5) " carolinianum L., wild geranium.

(5) Polygala polygama Walt., milkwort.

(I, 2, 3) Euphorbia polygonifolia L., seaside spurge.

(5) " corollata L., flowering spurge.

(7) Impatiens biflora Walt., jewelweed.

(7) “ pallida Nutt., jewelweed or touch-me-not $(u)$.

$(4,5)$ Hypericum Kalmianum L., Kalm's St. John's-wort.

(8) “ prolificum L., shrubby St. John's-wort.

(5) Hudsonia tomentosa Nutt.

(5) Helianthemum canadense Michx., frost weed.

(5*) Viola pedata L., bird-foot violet.

(5) " sagittata Ait., arrow-leaved violet.

$(6,7)$ " canadensis L., Canada violet.

$(6,7)$ " cucullata Ait., blue violet $(u)$.

$(6,7)$ " rostrata Pursh, long-spurred violet.

(6) " palmata L., palmate blue violet $(u)$.

(6) " conspersa Reich., dog violet $(u)$.

(5) Opuntia Rafinesquii Engelm., prickly pear.

$(2,5)$ Oenothera biennis L., common evening primrose.

$(2,5) \quad$ " rhombipetala Nutt., evening primrose.

(7) Circaea lutetiana L., enchanter's nightshade.

$(5,6)$ Aralia nudicaulis L., wild sarsaparilla.

(7) Panax quinquefolium L., ginseng.

$(6,7)$ " trifolium L., dwarf ginseng.

(7) Erigenia bulbosa Nutt., har inger of spring $(u)$.

(6) Taenidia integerrima Drude, yellow pimpernel $(u)$. 
(7) Sanicula marilandica L., sanicle.

(7) Cryptotaenia canadensis DC., honewort.

$(5,6,7)$ Monotropa uniflora L., Indian pipe.

(4) Chimaphila umbellata Nutt., prince's pine.

$(4,6)$ Pyrola elliptica Nutt., shin leaf.

(4) " secunda L., shin leaf.

(5) Epigaea repens L., May flower; trailing arbutus.

$(4,5)$ Gaultheria procumbens L., aromatic wintergreen.

(8) Sabbatia angularis Pursh.

(8) Gentiana crinita Froel., fringed gentian.

(8) " Andrezesii Griseb., closed gentian.

(6) A pocynum androsaemifolium L., dogbane (u).

(5) Asclepias tuberosa L., butterfly weed.

$(2,3,5)$ " syriaca L., common milkweed.

(7). “ phytolaccoides Pursh, poke milkweed.

(5) Acerates viridiflora Ell., green milkweed.

$(5,6)$ Cuscuta Gronovii Willd., dodder.

$(4,5,6)$ Phlox pilosa L., hairy phlox.

$(6,7)$ " divaricata L., blue phlox.

$\left(5^{*}\right)$ " bifida Beck., cleft phlox.

(6) Polemonium reptans L., Jacob's ladder $(u)$.

(7) Hydrophyllum appendiculatum Mich., waterleaf.

(6) Mertensia virginica Link., bluebells or Virginian cowslip $(u)$.

(6) Myosotis virginica BSP., small white forget-me-not.

$(4,5)$ Lithospermum canescens Lehm., puccoon.

$\left(4^{*}, 5\right) \quad$ "Gmelini Hitchc., puccoon.

(5) Monarda fistulosa L., wild bergamot.

$(4,5)$ " punctata L., horse-mint

(6) Hedeoma pulegioides Pers., American pennyroyal.

$(3,5)$ Physalis lanceolata Michx., ground-cherry.

$(3,5) \quad$ " virginiana Mill., ground-cherry.

(5) Linaria canadensis Dumont., toadflax.

(6) Collinsia verna Nutt., blue-eyed Mary (u).

(5) Gerardia pedicularia L., false foxglove.

(8) " purpurea L., purple gerardia.

(8) Castilleja coccinea Spreng., painted-cup.

(5) Pedicularis canadensis L., lousewort.

(6) Melampyrum lineare Lam., cow wheat.

(8) Utricularia cornuta Michx., small bladderwort.

(7) Epifagus virginiana Wallr., beech-drops.

(6) Conopholis americana Wallr., cancer-root.

$(2,3)$ Orobanche fasciculata Nutt., yellow cancer-root.

(7) Galium A parine L., bed-straw, cleavers.

$(6,7)$ " circaezans Michx., wild liquorice.

$(6,7)$ " concinnum T. \& G., shining bedstraw.

$(6,7)$ Mitchella repens L., partridge berry.

(6) Houstonia caerulea L., bluets (u).

$(4,5)$ Campanula rotundifolia L., bluebell.

(4) Linnaea borealis americana Rehder., twin-flower. 
(6) Triosteum perfoliatum L., horse gentian.

(7) Eupatorium urticaefolium Reichard, white thoroughwort.

(5) Liatris cylindracea Michx., blazing-star.

(5) " scariosa Willd., blazing-star.

$(4,5)$ Aster sericeus Vent.

$(4,5)$ " linariifolius $\mathrm{L}$., narrow-leaved aster.

(8) " azureus Lindl., blue aster.

(7) " cordifolius L., heart-leaved aster.

(6) “ macrophyllus L., heart-leaved aster.

(6) Erigeron pulchellus Michx., Robin's plantain.

(5) Solidago speciosa Nutt., goldenrod.

$\left(2^{*}, 3^{*}\right)$ " racemosa Gillmani Fernald, goldenrod.

(5) " nemoralis Ait., goldenrod.

$(2,3)$ " rigida L., goldenrod.

$\left(5^{*}\right) \quad$ " hispida Muhl., rough goldenrod.

$(6,7)$ " caesia L., goldenrod.

(5) Antennaria plantaginifolia Richards, everlasting.

(I) Xanthium canadense Mill., cockle-bur.

(8) Rudbeckia hirta L., yellow daisy.

(5) Helianthus divaricatus L., sunflower.

(5) " occidentalis Riddell, sunflower.

(4) Coreopsis palmata Nutt., stiff tickseed.

(4) “ lanceolata L., lance-leaved tickseed.

(8) " tripteris L., tall coreopsis.

( $1,2,3,4)$ Artemisia caudata Michx., wormwood.

$(\mathrm{I}, 2,3,4) \quad$ " canadensis Michx., wormwood.

$(\mathrm{I}, 2,3)$ Cirsium Pitcheri T. \& G., sand-thistle.

$(4,5)$ Krigia virginica Willd., dwarf dandelion.

$(5,6)$ “ amplexicaulis Nutt., dwarf dandelion.

$(6,7)$ Prenanthes alba L., rattlesnake root.

(7) Polymnia uvedalia L., leafcup.

(5) Hieracium canadense Michx., Canada hawkweed.

\section{THE PRAIRIE ASSOCIATIONS}

Shallow lakes or marshes have apparently one of two destinies, namely, either a forest or a prairie. East of Chicago the former succession is almost universal, but both are to be seen in this region. The stages in plant succession are not always clearly in evidence upon the prairie, but in the following list of species those of the low prairie (I), usually comprising a Spartina and a Calamagrostis association, are distinguished from those more characteristic of the Panicum and the PoaAndropogon associations of the higher prairie (2). HARVEY has shown (Trans. Ill. Acad. Sci. 6:1913) the average evaporation in a prairie association to be about equal to that of the oak dune and the water content of the soil to be decidedly low during the summer months. Comparatively undisturbed prairie areas are to be seen at Chicago Lawn and Ashburn, Ill. 
(2) Andropogon scoparius Michx., blue-stem grass.

$\left(2^{*}\right)$ " furcatus Muhl., blue-stem grass.

(I) Sorghastrum nutans Nash., Indian grass.

(2*) Panicum virgatum L., switch grass.

(2) Sporobolus heterolepis Gray, drop-seed grass.

(2) Agrostis alba L., red top grass.

( ${ }^{*}$ ) Calamagrostis canadensis Beauv., blue-joint grass.

(2) Koeleria cristata Pers.

(2) Danthonia spicata Beauv., wild oat grass.

(I*) Spartina Michauxiana Hitch., cord grass.

(2*) Poa compressa L., Canada blue grass.

(2) - " pratensis L., Kentucky blue grass.

$\left(\mathrm{I}^{*}\right)$ Glyceria nervata Trin., fowl meadow grass.

(2) Festuca elatior L., meadow fescue grass.

(2) Bromus Kalmii Gray, wild chess.

(2) Hordeum jubatum L., squirrel-tail grass.

(2) Elymus canadensis L., wild rye.

( $\left.{ }^{*}\right)$ Eleocharis palustris R. \& S., spike rush.

(I) Cyperus strigosus L.

(I) Fimbristylis castanea Vahl.

(I) Scirpus lineatus Michx.

(I) " fluviatilis Gray, bulrush.

(I) Carex scoparia Schkuhr.

(I) " cristata Schwein.

(I) " Sartwellii Dewey.

(I) " stricta Lam.

(I) " lanuginosa Michx.

(I) " straminea Willd.

(I) Juncus nodosus L., rush.

(I) " Torreyi Coville, Torrey's rush.

(I) “ acuminatus Michx., rush.

(2*) Allium cernuum Roth., wild onion.

(2) Lilium philadelphicum L., orange lily.

(2) " canadense L., wild yellow lily.

(2) Camassia esculenta Robinson, wild hyacinth.

(I) Iris versicolor L., blue flag.

(2) Sisyrinchium angustifolium Mill., blue-eyed grass.

(2) Comandra umbellata Nutt., bastard toad-flax.

(2) Silene antirrhina L., sleepy catchfly.

(2) Heuchera hispida Pursh, alum root.

(2) Stellaria longifolia Muhl., long-leaved chickweed.

(2) Thalictrum dasycarpum Fisch \& Lall., meadow rue.

(2) Potentilla canadensis L., Canada cinquefoil.

(2) " argentea L., silvery cinquefoil.

(2) " arguta Pursh, rough cinquefoil.

(2) Fragaria virginiana Duchesne, wild strawberry.

(2) Amorpha fruticosa L., false indigo.

(2) " canescens Pursh, lead-plant.

(2) Petalostemum purpureum Rydb., purple prairie clover. 
(2) Petalostemum candidum Michx., white prairie clover.

(I) Lathyrus pahustris L., marsh-pea.

(2) Desmodium illinoense Gray, tick-trefoil.

(I) Polygala sanguinea L., milkwort.

(I) Hypericum virgatum Lam., St. John's-wort.

(I) " mutilum L., St. John's-wort.

(I) Viola papilionacea Pursh, violet.

(2) " sagittata Ait., sagittate violet.

(I) “ pedatifida G. Don., prairie violet.

(I) Lythrum alatum Pursh, winged loosestrife.

(I) Oenothera pratensis Robinson, sun-drops.

(2) Gaura biennis L.

(2) Zizia aurea Koch., golden Alexanders.

(2) Eryngium yuccifolium Michx., button snakeroot.

(I) Cicuta maculata L., water-hemlock.

(I) Oxypolis rigidior (L.) Coult. \& Rose., cowbane.

(2) Dodecatheon Meadia L., shooting-star.

(2) Lithospermum canescens Lehm., puccoon.

(2) Satureja glabra Fernald, calamint.

(I) Steironema quadriflorum Hitch., loosestrife.

(I) " lanceolatum Gray, loosestrife.

(2) Gentiana puberula Michx., gentian.

(2) “ flavida Gray, gentian.

(2) A pocynum cannabium L., Indian hemp.

(I) Asclepias incarnata L., swamp milkweed.

(I) “ Sullivantii Englem., Sullivant's milkweed.

(2) " verticillata L., verticillate milkweed.

(2) Acerates floridana Hitch., green milkweed.

(I) Convolvulus sepium L., hedge bindweed.

(2) Phlox glaberrima L., smooth phlox.

(2) " pilosa L., hairy phlox.

(2) Cynoglossum officinale L., hound's-tongue.

(2) Verbena stricta Vent., hoary vervain.

(2) Scutellaria parvula Michx., small skullcap.

(2) Physostegia virginiana Benth., dragon-head.

(I) Stachys palustris L., woundwort.

(2) Pycnanthemum virginianum L., mountain mint.

(2) " flexuosum BSP., mountain mint.

(I) Lycopus americanus Muhl., water horehound.

(2) Chelone glabra L., snake's-head.

(2) Veronica virginica L., Culver's root.

(2) Gerardia purpurea L.

(2) " tenuifolia Vahl.

(I) Pentstemon laevigatus Ait., beard-tongue.

(I) Mimulus ringens L., monkey flower.

(2) Galium boreale L., northern bedstraw.

(I) " tinctorium L., bedstraw.

(I) Campanula aparinoides Pursh, marsh bellflower.

(2) Lobelia spicata Lam., wild lobelia. 
(2) Vernonia fasciculata Michx., ironweed.

(2) " altissima Nutt., ironweed.

(2) Kuhnia eupatorioides L., false boneset.

(2) Liatris spicata Willd., blazing star.

(2) " cylindracea Michx., blazing star.

(2) " scariosa Willd, button snakeroot.

(I) Boltonia asteroides L'Her., aster-like Boltonia.

(2) Solidago rigida L., rigid goldenrod.

(2) " canadensis L., Canada goldenrod.

(2) " serotina Ait., late goldenrod.

(I) " Riddellii Frank, goldenrod.

(2) " graminifolia Salisb., grass-leaved goldenrod.

(2) Aster novae-angliae L., New England aster.

(2) " multiflorus Ait., many-flowered aster.

$\left(2^{*}\right)$ " vimineus Lam., many-flowered aster.

(2) " paniculatus Lam., panicled aster.

(2) " ericoides L., frost-weed aster.

(2) Erigeron philadelphicus L., fleabane.

(2) " ramosus $\mathrm{BSP}$.

(2) Antennaria plantaginifolia Rich., plantain-leaved everlasting.

(2) Silphium laciniatum L., compass-plant.

(2) " terebinthinaceum Jacq., prairie-dock.

(2) " integrifolium Michx., rosinweed.

(2) Parthenium integrifolium L., feverfew.

(I) Rudbeckia triloba L., thin-leaved cone-flower.

(2) " hirta L., black-eyed-Susan.

(2) Heliopsis helianthoides Sweet, ox-eye.

(2) Lepachys pinnata T. \& G., cone flower

(2) Helianthus strumosus L., sunflower.

(2) " grosseserratus Martens, sunflower.

(2) " scaberrimus Ell., sunflower.

(2) Cacalia tuberosa Nutt., Indian plantain.

(I) Senecio aureus L., golden ragwort.

(I) " Balsamitae Muhl., golden ragwort.

(2) Cirsium virginianum (L.) Michx., thistle.

(I) " altissimum (L.) Spreng., thistle.

(2) " pumilum Spreng., pasture thistle.

(2) " lanceolatum Hill., bull thistle.

(2) Krigia amplexicaulis Nutt., Virginia goatsbeard.

(2) Lactuca canadensis L., wild lettuce.

(2) “ ludoviciana Riddell, wild lettuce.

\section{AQUATIC ASSOCIATIONS}

These associations reach their best development in the shallow lakes and ponds of the old lake plain and in the depressions in the sand dune area. Others are found in connection with the development of the stream system. The succession in these ponds, streams, and swamps 
may lead to either a grassland or a forest. Only seed plants and ferns are included in the following lists which are classified to indicate the (I) submerged, (2) floating-leaved, and (3) emergent aquatics, the (4) reed, and the (5) sedge swamp associations. Subsequent stages in the successions are given under the prairie, peat bog, and flood plain associations. Among the many localities in which aquatic vegetation is to be found some of the best are at Miller, Long Lake, Pine, Wolf, and Calumet lakes, Ind., Skokie Marsh and Fox Lake, Ill.

\section{Shrubs}

(3) Salix amygdaloides Anders., peach-leaved willow.

(3) " cordata Muhl., heart-leaved willow.

(3) " glaucophylla Bebb, glaucous willow.

(3) " longifolia Muhl., sand-bar willow.

(3) " discolor Muhl., glaucous willow.

(3) Cephalanthus occidentalis L., buttonbush.

\section{Herbaceous Plants}

(5) Selaginella apus Spreng., marsh selaginella.

(3) Equisetum variegatum Schleich., variegated horsetail.

(3) Equisetum fluviatile L., swamp horsetail.

(4*) Typha latifolia L., common cat-tail.

(4*) " angustifolia L., narrow-leafed cat-tail.

(3*) Sparganium eurycarpum Engelm., bur-reed.

$\left(2^{*}\right)$ Potamogeton natans L., pondweed.

(2) " americanus C. \& S., American pondweed.

(2) " amplifolius Tuckerm., broad-leaved pondweed.

$\left(I^{*}\right) \quad$ " crispus L., crisp pondweed.

(I) " lucens L., shining pondweed.

$\left(I^{*}\right) \quad$ zosterifolius Schu., grass-leaved pondweed.

(I*) " pectinatus L., narrow-leaved pondweed.

(I) “ Robbinsii Oakes, Robbin's pondweed.

(I) Zannichellia palustris L., horned pondweed.

(I*) Najas flexilis R. \& S., naiad.

(4) Triglochin maritima L., arrow grass.

$\left(3^{*}\right)$ Sagittaria latifolia Willd., arrow-head.

(3) " heterophylla Pursh, arrowweed.

(3) " graminea Michx., narrow arrow-head.

(3) Alisma plantago-aquatica L., water plantain.

( $\left.{ }^{*}\right)$ Elodea canadensis Michx., water-weed.

(I) Vallisneria spiralis L., tape grass.

$(3,5)$ Calamagrostis canadensis Beauv., blue-joint grass.

(4*) Phragmites communis Trin., reed.

(5) Spartina Michauxiana Hitch., cord grass.

(3) Zizania aquatica L., wild rice.

(3) Eleocharis palustris R. \& S., spike rush.

temuis Schultes, spike rush. 
(5) Eleocharis acicularis R. \& S., spike rush.

(3*) Scirpus validus Vahl., great bulrush.

$\left(3^{*}\right)$ " fluviatilis Gray, river bulrush.

$(4,5)$ " americanus Pers., triangular bulrush.

(2) Dulichium arundinaceum Britton.

( $\left.{ }^{*}\right)$ Carex lanuginosa Michx., woolly sedge.

(5) " filiformis L., slender sedge.

(5) " aurea Nutt., golden sedge.

$\left(5^{*}\right)$ " stricta Lam., narrow sedge.

(5) " spp., sedges.

(5) Rynchospora capillacea Torr., beak rush.

(5) Cladium mariscoides Torr., twig rush.

(3) Symplocarpus foetidus Nutt., skunk cabbage.

$(3,5)$ Peltandra virginica Knuth., arrow arum.

(4) Acorus calamus L., sweet flag.

(2*) Spirodela polyrhiza Schleid., duckweed.

( $\left.{ }^{*}\right)$ Lemna trisulca L., duckweed.

$\left(2^{*}\right)$ " minor L., duckweed.

(2) Wolffia columbiana Karst., smallest duckweed.

(3*) Pontederia cordata L., pickerel-weed.

(3) Heteranthera dubia MacM., water star-grass.

(5) Juncus nodosus L., knotted rush.

(5) " canadensis Gay., Canada rush.

(5) " balticus Willd., Baltic rush.

(3, 5) Iris versicolor L., blue flag.

(3) Saururus cernuus L., lizard's tail.

$(\mathbf{I}, 3,5)$ Polygonum amphibium L., water smartweed.

$(\mathrm{I}, 3,5) \quad$ " Muhlenbergii Wats., water smartweed.

$(3,5) \quad$ " hydropiperoides Michx., water pepper.

( $\left.{ }^{*}\right)$ Ceratophyllum demersum L., hornwort.

(2*) Nymphaea advena Ait., yellow pond lily.

$\left(2^{*}\right)$ Castalia tuberosa Greene, white water lily.

(2*) Brasenia Schreberi Gmel., water shield.

(2) Nelumbo lutea Pers., American lotus or nelumbo.

(I) Ranunculus aquatilis L., white water buttercup.

(I) " delphinifolius Torr., yellow water buttercup.

(5) " pennsylvanicus L., bristly buttercup.

(3) Caltha palustris L., marsh marigold.

$(\mathrm{I}, 3)$ Radicula Nasturtium-aquatica B. \& R., water cress.

(I) “ aquatica Rob., lake cress.

(3) " palustris Moench., marsh cress.

(3) Cardamine hirsuta L., bitter cress.

$(3,5)$ Penthorum sedoides L., ditch stonecrop.

(3) Potentilla palustris Scop., marsh cinquefoil.

(2) Callitriche palustris L., water starwort.

(5) Hypericum virginicum L., marsh St. John's-wort.

(3) “ canadense L., Canada St. John's-wort.

(5) Lythrum alatum Pursh, winged loosestrife.

$(3,5)$ Ludvigia palustris Ell., water purslane. 
(5) Epilobium molle Torr., willow-herb.

( $\left.{ }^{*}\right)$ Myriophyllum spicatum L., water milfoil.

$\left(\mathrm{I}^{*}\right) \quad$ " heterophyllum Michx., water milfoil.

(3) Proserpinaca palustris L., mermaid-weed.

(3) Hippuris vulgaris L., mare's tail.

$(3,5)$ Sium cicutaefolium Schrank., water parsnip.

$(3,5)$ Cicuta bulbifera L., bulb-bearing water hemlock.

(5) " maculata L., water hemlock.

(5) Lysimachia thyrsiflora L., tufted loosestrife.

(5) " terrestris BSP., bulbous loosestrife.

(3) Menyanthes trifoliata L., buckbean.

(5) Asclepias incarnata L., swamp milkweed.

(5) Verbena hastata L., blue vervain.

(5) Scutellaria galericulata L., marsh skullcap.

(5) Lycopus americanus Muhl., water horehound.

(5) " virginicus L., bugle weed.

(5) Mentha arvensis canadensis L., Canada mint.

(3) Veronica Anagallis-aquatica L., water speedwell.

( $\left.{ }^{*}\right)$ Utricularia vulgaris L., bladderwort.

(I) " minor L., smaller bladderwort.

(I) Dianthera americana L., water willow.

(3) Plantago cordata Lam., river plantain.

(5) Galium asprellum Michx., marsh bedstraw.

$(3,5)$ Campanula aparinoides Pursh., marsh bellflower.

(5) Eupatorium purpureum L., Joe-Pye weed.

(5) " perfoliatum L., boneset or thoroughwort.

(5) Solidago graminifolia Salisb., grass-leaved goldenrod.

(5) Cirsium muticum Michx., swamp thistle.

(5) Bidens trichosperma Britton, marsh stick-tight.

(5) " frondosa L., beggar-tick.

\section{THE PEAT BOG ASSOCIATIONS}

The associations in the peat bog series that are best developed are (I) the sedge, (2) the xerophytic shrub, (3) the tamarack, and (4) the pine-birch forest associations. No separate discussion of the stages in the succession will be attempted but the association in which the following species occur most abundantly will be indicated. These associations are seen at Mineral Springs and Mill Creek, Ind., and Fox Lake, Ill.

\section{Trees and Shrubs}

(4*) Pinus Strobus L., white pine.

(4) Thuja occidentalis L., arbor vitae.

(3*) Larix laricina Koch., tamarack or American larch.

$(\mathrm{I}, 2)$ Salix pedicellaris Pursh.

$(\mathrm{I}, 2)$ " candida Flugge, hoary willow.

(2) " discolor Muhl., glaucous willow. 
(4) Betula lutea Michx., yellow birch.

(4) “ pumila L., dwarf birch.

$(2,3)$ Almus incana Moench., hoary alder.

(4) Ulmus americana L., American elm.

$(3,4)$ Rubus hispidus L., low swamp blackberry.

$(3,4)$ " villosus Ait., dewberry.

$(3,4)$ " triflorus Richards, dwarf raspberry.

$(3,4)$ " idaeus L., red raspberry.

$\left(2^{*}\right)$ Rosa carolina L., swamp rose.

(4) Pyrus arbutifolia L., chokeberry.

(4) Amelanchier canadensis Medic., service-berry.

(2*) Rhus Vernix L., poison-sumach.

$(2,3)$ Ilex verticillata Gray, northern-holly.

$(2,3)$ Nemopanthus mucronata Trel., mountain-holly.

(4*) Acer rubrum L., red maple.

$(2,3)$ Rhamnus alnifolia L'Her., swamp buckthorn.

(4) Tilia americana L., basswood or American linden. .

(3*) Chamaedaphne calyculata Moench., cassandra.

(3) Andromeda polifolia L., Andromeda.

$(3,4)$ Vaccinium corymbosum L., tall blueberry.

(I) " macrocarpon Ait., cranberry.

$(2,3)$ Cornus stolonifera Michx., red-osier dogwood.

(3) " canadensis L., dwarf dogwood.

(4) Nyssa sylvatica Marsh., sour gum.

(4) Sambucus racemosa L., red'berried elder.

(4) Lonicera dioica L., swamp honeysuckle.

\section{Herbaceous Plants}

$(3,4)$ Osmunda regalis L., royal fern.

$(3,4)$ " Claytoniana L., interrupted fern.

$(3,4)$ " cinnamomea L., cinnamon fern.

$\left(3^{*}, 4\right)$ Onoclea sensibilis L., sensitive fern.

$\left(\mathrm{I}^{*}, 2\right)$ Aspidium Thelypteris Sw., swamp fern.

$(3,4) \quad$ " cristatum Sw., cristate shield fern.

(2*) Woodwardia virginica Sm., chain fern.

(I) Glyceria nervata Trin., manna grass.

(I) Zizania aquatica L., wild rice.

(I) Dulichium arundinaceum Britton.

(I*) Eleocharis rostellata Torr., spike-rush.

(I) “ palustris (L.) R. \& S., spike-rush.

(I) Scirpus validus Vahl., great bulrush.

(I) " cyperinus Kunth., wool grâss.

(I) " atrovirens Muhl., dark green bulrush.

(I) Cyperus strigosus L., straw-colored cyperus.

(I) " erythrorhizos Muhl.

(I) " Engelmanni Steud.

(I) Eriophorum virginicum L., cotton grass.

(I) Cladium mariscoides Torr., twig-rush. 
(I) Scleria triglomerata Michx., nut-rush.

(I) Rynchospora glomerata (L.) Vahl., beak-rush.

(3) Carex trisperma Dewey, soft-leaved sedge.

$(\mathrm{I}, 3)$ " stellulata Good, prickly sedge.

(I) " tenella Schkuhr., sedge.

(I) " crinita Lam., fringed sedge.

(I) " filiformis L., slender sedge.

(I) " Pseudo-Cyperus L., cyperus sedge.

(I) Juncus tenuis Willd., slender rush.

$(3,4)$ Maianthemum canadense Desf., wild lily-of-the-valley.

$(\mathrm{I}, 2)$ Iris versicolor $\mathrm{L}$., blue flag.

(3) Cypripedium parviflorum Salisb., yellow lady's slipper.

(2) " hirsutum Mill., showy lady's slipper.

$(\mathrm{I}, 2)$ " candidum Muhl., white lady's slipper.

(4) “ acaule Ait., stemless lady's slipper.

(I, 3) Habenaria hyperborea (L.) R.Br., rein orchis.

$(\mathrm{I}, 3) \quad$ " clavellata Spreng., rein orchis.

$(\mathrm{I}, 3) \quad$ " ciliaris $\mathrm{R}$. Br., fringed orchis.

(I) " blephariglottis Torr., white fringed orchis.

(I) " psycodes Sw., purple fringed orchis.

(I) Calopogon pulchellus R.Br.

(I) Spiranthes cernua (L.) Richards, ladies' tresses.

(3) Pilea pumila Gray, clear weed.

(1) Boehmeria cylindrica Sw., false nettle.

(2) Thalictrum dasycarpum F. \& L., meadow rue.

(I) Sarracenia purpurea L., pitcher-plant.

(2) Drosera rotundifolia L., sundew.

(2) " longifolia L., sundew.

(3) Saxifraga pennsylvanica L., swamp saxifrage.

(3) Chrysosplenium americanum Schwein., golden saxifrage.

(I) Parnassia caroliniana Michx., grass of Parnassus.

$(3,4)$ Coptis trifolia Salisb., goldthread.

(2) Spiraea latifolia Borkh., meadow-sweet.

(2) " tomentosa L., hardhack.

(I) Viola lanceolata L., lance-leaved violet.

(I) " pallens Brainerd, sweet white violet.

(4) Aralia nudicaulis L., wild sarsaparilla.

$\left(\mathrm{I}^{*}, 2\right)$ Decodon verticillatus Ell., water willow.

(4) Trientalis americana Pursh, star-flower.

(3, 4) Galium triflorum Michx., sweetscented bedstraw.

(4) Mitchella repens L., partridge berry.

(3) Myosotis laxa Lehm., forget-me-not.

(3) Linnaea borealis L., twinflower.

(I) Solidago patula Muhl., bog goldenrod.

(I) " neglecta T. \& R., bog goldenrod.

(I) Aster puniceus L., bog aster.

(I) Bidens trichosperma Britton, tickseed. 


\section{THE ROCK RAVINE ASSOCIATIONS}

Well-developed narrow rock ravines with nearly perpendicular sides are so well shaded and the air currents are so reduced that the evaporating power of the air is reduced to a minimum. This permits the development of associations of liverworts, mesophytic mosses, delicate ferns, and extreme shade plants such as Impatiens and Pilea. Upon ledges along the sides of the canyons and at the foot of the walls as soon as sufficient soil accumulates a herbaceous and shrub vegetation similar to the undergrowth of the climax mesophytic forest (q.v.) makes its appearance. With the widening of the canyon and the accumulation of talus the trees of the same forest appear, while as the floor of the canyon widens the vegetation of the flood plain (q.v.) develops upon it. The upper edges of the ravine have a vegetation similar to that of the river bluff (q.v.).

In the following lists only the more characteristic species of some small limestone canyons near Sag and Lemont, Ill., and of the large sandstone canyons of Starved Rock Park are included. No attempt will be made to list the mosses or leafy liverworts, but the thallose liverworts include: Riccia natans, Marchantia polymorpha, Reboulia sp., Aneura pinguis, Pellia epiphylla, Blasia pusilla, Scapania (nemorosa?), Cephalozia sp., and Anthoceros laevis.

\section{Trees and Shrubs}

Taxus canadensis Marsh., American yew.

Asimina triloba Dunal., American papaw.

Hydrangea arborescens L., wild hydrangea.

Ribes Cynosbati L., gooseberry.

Aruncus sylvester Kosteletzsky, goat's-beard.

\section{Herbaceous Plants}

Phegopteris polypodioides Fee, beech-fern.

Asplenium angustifolium Michx., narrow-leaved spleenwort.

Cystopteris bulbifera (L.) Bernh., bulb-bearing fern.

“ fragilis (L.) Bernh., fragile fern.

Cryptogramma Stelleri Prantl, slender cliff-brake.

*Pilea pumila (L.) Gray, clearweed.

Parietaria pennsylvanica Muhl., pellitory.

Saxifraga pennsylvanica L., swamp saxifrage.

*Arabis brachycarpa Britton, rock cress.

“ laevigata Poir., rock cress.

Impatiens biflora Walt., spotted touch-me-not.

Aralia racemosa L., wild spikenard.

Solidago latifolia L., broad-leaved goldenrod. 


\section{THE RIVER CLIFF ASSOCIATIONS}

With the exception of a few poorly developed limestone cliffs along the Des Plaines River and its tributaries there are no rock cliffs in the immediate Chicago region. High limestone cliffs are to be seen on the Mississippi at Savanna, Ill., while at Starved Rock Park are cliffs consisting of rather easily eroded sandstone which permits the rapid development of mosses and seed plants but is unfavorable to the growth of lichens. Among the mosses several species of Polytrichum (e.g., P. juniperinum and $P$. piliferum) are prominent. In even slightly protected situations a layer of sandy soil soon accumulates, hence it is not surprising that upon level and gently sloped areas a vegetation similar to the oak associations of the sand dunes (q.v.) soon becomes established. Many of the plants in the following list are quite as characteristic of river banks of clay as of the rock cliffs.

Pinus Strobus L., white pine.

Trees and Shrubs

Thuja occidentalis L., arbor vitae.

*Juniperus virginiana L., red cedar.

Populus tremuloides Michx., American aspen.

“ grandidentata Mich., large-toothed aspen.

*Ostrya virginiana K. Koch., hop-hornbeam.

Carpinus caroliniana Walt., water-beech.

Ribes Cynosbati L., prickly gooseberry.

*Physocarpus opulifolius Maxim., nine-bark.

Crataegus tomentosa L., red haw.

“ spp., haws or thorn apples.

Amelanchier canadensis (L.) Medic., service-berry.

Prunus virginiana L., chokecherry.

Pyrus arbutifolia L., chokeberry.

Ptelea trifoliata L., hop tree.

Rhus glabra L., smooth sumach.

* " typhina L., rough sumach.

" Toxicodendron L., poison-ivy.

Ilex verticillata (L.) Gray, northern holly.

Celastrus scandens L., climbing bittersweet.

Vaccinium pennsylvanicum Lam., blueberry.

" canadense Kalm., blueberry.

*Lonicera Sullivantii Gray, Sullivant's honeysuckle.

*Viburnum prunifolium L., plum-leaved haw.

\section{Herbaceous Plants}

Selaginella rupestris (L.) Spring.

Polypodium vulgare L., polypody fern.

Pellaea atropurpurea Link., purple cliff-brake.

Pteris aquilina L., common brake. 
Camptosorus rhizophyllus Link., walking fern.

Panicum dichotomum L., prairie-grass.

Cenchrus tribuloides L., sand-bur.

Poa compressa L., wire-grass.

Festuca octoflora Walt., fescue-grass.

Hordeum jubatum L., squirrel-tail grass.

Carex Muhlenbergii Schkuhr.

Cerastium nutans Raf., chickweed.

Talinum teretifolium Pursh, flame flower.

Heuchera hispida Pursh, alum-root.

Ranunculus fascicularis Muhl., early fascicled buttercup.

Aquilegia canadensis L., columbine.

Draba caroliniana Walt., spring draba.

Vicia caroliniana Walt., pale vetch.

“ americana Muhl., American vetch.

Amorpha canescens Pursh, lead-plant.

Oxalis stricta L., yellow wood sorrel.

" violacea $\mathrm{L}$., violet wood sorrel.

Polygala Senega L., Seneca snakeroot.

Taenidia integerrima Drude, yellow pimpernel.

Dodecatheon Meadia L., shooting star.

Asclepias verticillata $\mathrm{L}$., verticillate milkweed.

Verbena angustifolia Michx., narrow-leaved vervain.

“ stricta Vent., hoary vervain.

Scutellaria parvula Michx., small skullcap.

Linaria canadensis (L.) Dumont., toadflax.

Pentstemon hirsutus Willd., hairy beard-tongue.

Orobanche uniflora L., cancer root.

Houstonia caerulea L., bluets.

Myosotis virginica (L.) BSP., white forget-me-not.

Specularia perfoliata (L.) A. DC., Venus' looking-glass.

Campanula rotundifolia $\mathrm{L}$., harebell.

Solidago nemoralis Ait., goldenrod.

Aster ptarmicoides T. \& G., upland white aster.

Antennaria plantaginifolia (L.) Richards, everlasting.

Brauneria angustifolia Heller, purple coneflower.

Achillea Millefolium L., yarrow.

\section{THE FLOOD-PLAIN ASSOCIATIONS}

From the nature of their physiography there are many species common to the climax mesophytic forest (q.v.) and the fairly mature flood plain. The earlier stages of the latter are characterized by a number of rapidly growing trees and a herbaceous undergrowth of great luxuriance, in which the broad "shade leaf" is seen at its highest development. Nowhere is the development of climbing and twining plants so abundant. The different stages in the development of the flood-plain vegetation are not distinguished in the following list of species. In the list are 
included plants of depressions in the uplands that give rise to swampy conditions, while many plants of the water's edge will be found in the list of aquatics (q.v.). Swampy depressions in the morainic uplands are common throughout the moraine deposits, and some of the best examples of flood-plain associations may be seen along the Galien River at Three Oaks, Mich., along the Illinois River at Starved Rock Park, along the Des Plaines River at Riverside and River Forest, and along: the Chicago River at Edgebrook.

\section{Trees and Shrubs}

*Salix nigra Michx., black willow.

" amygdaloides Anders., peach-leaved willow.

" longifolia Muhl., sand bar willow.

Populus deltoides Marsh., cottonwood.

Juglans cinerea L., butternut.

“ nigra L., black walnut.

Carya cordiformis K. Koch., bitter-nut hickory.

Quercus macrocarpa Michx., bur oak.

“ palustris Muench., pin oak.

“ bicolor Willd., swamp white oak.

Ulmus fulva Michx., slippery elm.

" americana L., American elm.

Celtis occidentalis L., hackberry.

Morus rubra L., red mulberry.

Menispermum canadense L., moonseed.

Platanus occidentalis L., sycamore.

Clematis virginiana L., virgin's-bower.

Benzoin aestivale Nees., spice bush.

Ribes Cynosbati L., prickly gooseberry.

" floridum L'Her., wild black currant.

${ }^{*}$ Crataegus punctata Jacq., punctate haw.

* " mollis Scheele., red haw.

“ spp., haws or thorn apples.

Pyrus coronaria L., American crab.

Prunus serotina Ehrh., wild black cherry.

" americana Marsh., wild plum.

Gleditsia triacanthos L., honey-locust.

Cercis canadensis L., redbud.

Gymnocladus dioica (L.) Koch., Kentucky coffee-tree.

Amorpha fruticosa $\mathrm{L}$.

Zanthoxylum americanum Mill., prickly ash.

Rhus Toxicodendron L., poison ivy.

Evonymus atropurpureus Jacq., waahoo or burning bush.

“ obovatus Nutt., strawberry-bush.

Staphylea trifolia L., bladdernut.

* Acer saccharinum L., white maple.

"Negundo L., box-elder. 
Vitis cordifolia Michx., frost-grape.

" vulpina L., river-bank grape.

Psedera quinquefolia (L.) Greene, Virginia creeper.

*Tilia americana L., basswood.

* Fraxinus americana $\mathrm{L}$., white ash.

" nigra Marsh., black ash.

" quadrangulata Michx., blue ash.

Sambucus canadensis L., common elder.

Viburnum Lentago L., sweet viburnum or black haw.

\section{Herbaceous Plants}

* Onoclea sensibilis L., sensitive fern.

" Struthiopteris Hoffm., ostrich fern.

Osmunda Claytoniana L., interrupted fern.

“ regalis $\mathrm{L}$., royal fern.

cinnamomea L., cinnamon fern.

Elymus virginicus L., wild rye.

*Arisaema triphyllum Schott., Indian turnip or Jack-in-the-pulpit.

" Dracontium Schott., green dragon.

Symplocarpus foetidus Nutt., skunk cabbage.

Uvularia grandiflora Sm., bellwort.

*Allium tricoccum Ait., wild leek.

" canadense L., wild onion or garlic.

Erythronium americanum Ker., yellow adder's tongue.

* “ albidum Nutt., white adder's tongue.

Trillium recurvatum Beck., wake-robin.

Polygonatum biflorum Ell., small Solomon's seal.

“ commutatum Dietr., great Solomon's seal.

Smilax herbacea L., carrion flower.

" hispida Muhl., cat-brier.

Dioscorea villosa L., wild yam.

Urtica gracilis Ait., nettle.

*Laportea canadensis (L.) Gaud., wood-nettle.

Pilea pumila (L.) Gray, clearweed.

Parietaria pennsylvanica Muhl., pellitory.

Asarum canadense L., wild ginger.

*Polygonum virginianum L., smartweed.

Polygonum scandens L., climbing false buckwheat.

Silene virginica L., fire pink.

" stellata Ait., starry campion.

*Claytonia virginica L., spring beauty.

Ranunculus septentrionalis Poir., early buttercup.

“ recurvatus Poir., hooked buttercup.

* “ abortivus L., abortive buttercup.

Clematis virginiana L., virgin's bower.

Isopyrum biternatum T. \& G.

Caltha palustris L., marsh marigold.

Caulophyllum thalictroides Michx., blue cohosh.

Dentaria laciniata Muhl., toothwort. 
Cardamine bulbosa BSP., spring cress.

* “ Douglasii Britton, spring cress.

Geum canadense Jacq., avens.

"Virginianum L., avens.

Desmodium grandiflorum DC., tick-trefoil.

Apios tuberosa Moench, groundnut.

Amphicarpa monoica Ell., hog peanut.

Floerkea proserpinacoides Willd., false mermaid.

Impatiens biflora Walt., jewel weed.

Hibiscus Moscheutos L., swamp rose mallow.

“ militaris Cav., halberd-leaved rose mallow.

*Viola cucullata Ait., blue violet.

* “ sororia Willd., blue violet.

“ pubescens Ait., yellow violet.

Circaea lutetiana L., enchanter's nightshade.

Aralia nudicaulis L., wild sarsaparilla.

* Sanicula marilandica L., sanicle.

Osmorhiza Claytoni Clarke, sweet Cicely.

“ longistylis DC., sweet Cicely.

${ }^{*}$ Cryptotaenia canadensis (L.) DC., honewort.

Heracleum lanatum Michx., cow-parsnip.

Chaerophyllum procumbens Crantz.

Angelica atropurpurea L., angelica.

Lysimachia Nummularia L., moneywort.

*Steironema ciliatum Raf., fringed loosestrife.

Asclepias phytolaccoides Pursh, poke milkweed.

Convolvulus sepium L., bindweed.

Ipomea pandurata L., man-of-the-earth.

Cuscuta Gronovii Willd., dodder.

*Phlox divaricata L., blue phlox.

Polemonium reptans L., Jacob's ladder.

Hydrophyllum macrophyllum Nutt., waterleaf.

" virginianum L., waterleaf.

“ appendiculatum Michx., waterleaf.

Ellisia Nyctelea L.

Phacelia bipinnatifida Michx.

Mertensia virginica Link., Virginia cowslip or bluebells.

Teucrium canadense L., American germander.

Scutellaria lateriflora L., skullcap.

Stachys palustris L., woundwort.

Lycopus virginicus L., bugleweed.

Sicyos angulatus L., bur-cucumber

Echinocystis lobata T. \& G., wild balsam-apple.

Campanula americana $\mathrm{L}$., tall bellflower.

Lobelia siphilitica L., great lobelia.

Eupatorium purpureum L., Joe-Pye weed.

Silphium perfoliatum L., cup-plant.

Ambrosia trifida L., great ragweed.

" artemisiifolia L., common ragweed. 
Rudbeckia laciniata L., wild goldenglow.

Helianthus tuberosus L., Jerusalem artichoke.

Cacalia atriplicifolia L., Indian plantain.

\section{THE LAKE CLIFF ASSOCIATIONS}

The shore of Lake Michigan, north of Chicago, exhibits a series of high clay cliffs cut in the moraine. These differ in slope according to the character of the soil and in their vegetation according to whether or not they are being attacked by waves at the present time. The earliest plant associations consist of the antecedent vegetation which has slumped from the upland together with shrubs and perennial herbs with means of rapid vegetative propagation which enable them to compete with the unstable substratum. Only the plants peculiar to the pioneer stages of the succession are included in the following list, as those of the beach and the mixed oak forest differ very little from corresponding associations upon the sand dunes (q.v.). The ravines opening upon the shore have the vegetation of the lake cliffs upon their exposed slopes and that of the mesophytic forest (q.v.) and flood plain (q.v.) upon the more protected areas.

\section{Trees and Shrubs}

Pinus Strobus L., white pine.

Juniperus virginiana L., red cedar.

" communis $\mathrm{L}$., common juniper.

Thuja occidentalis L., arbor vitae.

Salix discolor Muhl., pussy-willow.

" glaucophylla Bebb, glaucous willow.

Populus tremuloides Michx., aspen.

“ grandidentata Michx., large-toothed aspen.

“ balsamifera L., balsam poplar.

Corylus americana Walt., hazelnut.

Ostrya virginiana (Mill) K. Koch., hop-hornbeam.

Carpinus caroliniana Walt., ironwood.

Betula alba papyrifera Spach., white birch.

Amelanchier canadensis (L.) Medic., service-berry.

Rosa blanda Ait., wild rose.

" humilis Marsh., wild rose.

Prunus virginiana L., chokecherry.

Acer Negundo L., box-elder.

Rhus typhina L., staghorn sumach.

" glabra L., smooth sumach.

“ Toxicodendron L., poison-ivy.

Ceanothus americana L., New Jersey tea.

Shepherdia canadensis (L.) Nutt., buffalo-berry.

Elaeagnus argentea Pursh, silverberry.

Tilia americana L., basswood. 
Cormus paniculata L'Her., panicled dogwood.

“ stolonifera Michx., red-osier dogwood.

Viburnum Lentago L., black haw.

“ Opulus L., cranberry tree.

\section{Herbaceous Plants}

Equisetum arvense L., common horsetail.

" hyemale L., scouring-rush.

Poa compressa L., Canada blue-grass.

Elymus canadensis L., Canada rye grass.

Potentilla Anserina L., goose-grass.

" canadensis $\mathrm{L}$.

Melilotus alba Desr., sweet clover.

Astragalus canadensis L., milk vetch.

Vicia americana Muhl., purple vetch.

" caroliniana Walt., pale vetch.

Lathyrus ochroleucus Hook., pale vetchling.

Polygala Senega L., Seneca snakeroot.

Gentiana quinquefolia L., stiff gentian.

A pocynum androsaemifolium L., dogbane.

Asclepias syriaca L., common milkweed.

Aster laevis L., smooth aster.

“ multiflorus Ait., many flowered astèr.

Solidago arguta Ait., plume goldenrod.

Helianthus divaricatus L., sunflower.

Rudbeckia hirta L., black-eyed-Susan. 


HA 
(1)

1

(1)

(1)

10

(1)

(1)

(1)

(1)

40 (1)

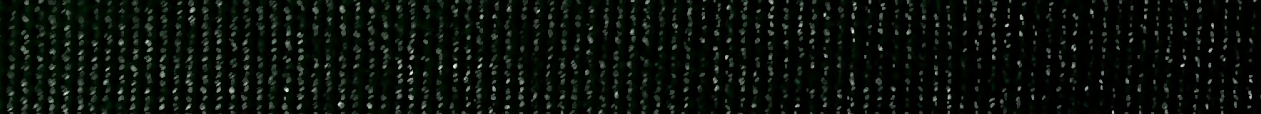

